\title{
Autologous PD-L1/CD80/CD86-targeted CAR-T Cells
}

National Cancer Institute

\section{Source}

National Cancer Institute. Autologous PD-L1/CD80/CD86-targeted CAR-T Cells. NCI

Thesaurus. Code C148216.

A preparation of autologous human T-lymphocytes eng ineered to express a chimeric antigen receptor (CAR) composed of a modified from of the human inhibitory receptor programmed cell death protein 1 (PD-1; PDCD1), in which the intracellular signal domain of PD-1 is transformed to allow for stimulatory signaling but with an intact extracellular ligand binding domain that specifically binds the tumor-associated antigen (TAA) programmed cell death-1 ligand 1 (PD-L1), and a modified form of the T-cell inhibitory receptor cytotoxic T-lymphocyte-associated antigen 4 (CT LA-4), with a transformed intracellular signal domain to allow for stimulatory signaling, which specifically binds the B7 proteins CD80 (B7-1) and CD86 (B7-2), with potential immunostimulating and antineoplastic activities. Usually, lig and binding to PD-1 and CT LA-4 inhibits T-cell activity; however, these modified forms of PD-1 and CT LA-4 promote T-cell stimulatory signaling. Upon administration, the autologous PD-L1/CD80/CD86-targeted CAR-T cells target and bind to PD-L1 expressed on certain tumor cells and to CD80/CD86 expressed on antigen-presenting cells (APCs). This stimulates T-cell activation, $\mathrm{T}$-cell proliferation and enhanced cytokine production, which induces selective toxicity in tumor cells expressing PD-L1. PD-1, found on activated T-cells, negatively regulates T-cell activity; it plays a key role in immune evasion and prevents tumor cell lysis. PD-L1 is often overexpressed on tumor cell types and plays a key role in immune evasion. The costimulatory molecules CD80 and CD86 play a key role in T-lymphocyte activation upon binding to CD28 upon antigen recog nition; however, binding of CD80 and CD86 to wildtype CT LA-4 inhibits T-cell activity and results in T-cell exhaustion. 